\title{
Density Exponent Analysis - A new vision towards gravitational collapse of molecular clouds
}

Guang-Xing Li ( $\sim$ gxli@ynu.edu.cn )

South-Western Institute for Astronomy Research, Yunnan University

Ji-Xuan Zhou

South-Western Institute for Astronomy Research, Yunnan University

Article

Keywords: molecular clouds, Density Exponent Analysis, gravitational collapse

Posted Date: July 29th, 2021

DOI: https://doi.org/10.21203/rs.3.rs-669614/v1

License: (c) (i) This work is licensed under a Creative Commons Attribution 4.0 International License. Read Full License 


\title{
Density Exponent Analysis - A new vision towards gravitational collapse of molecular clouds
}

\author{
Guang-Xing Li*1 and Ji-Xuan Zhou ${ }^{1}$ \\ ${ }^{1}$ South-Western Institute for Astronomy Research, Yunnan University, Kunming, \\ 650500 Yunnan, People's Republic of China; gxli@ynu.edu.cn
}

July 16, 2021

\begin{abstract}
The evolution of molecular interstellar clouds, during which stars form, is a complex, multi-scale process. The power-law density exponent describes the steepness of density profiles in the log-log space, and it has been used to characterize the density structures of the clouds. Its effectiveness results from the widespread emergence of power-law-like density structures in complex systems that have reached intermediate asymptotic states. However, its usage is usually limited to spherically symmetric systems. Importing the Level-Set Method, we develop a new formalism that generates robust maps of a generalized density exponent $k_{\rho}$ at every location for complex density distributions. By applying it to a high fidelity, high dynamical range map of the Perseus molecular cloud constructed using data from the Herschel and Planck satellites, we find that the density exponent exhibits a surprisingly wide range of variation $\left(-3.5 \lesssim k_{\rho} \lesssim-0.5\right)$. Regions at later stages of gravitational collapse are associated with steeper density profiles. Inside a region, gas located in the vicinities of dense structures has very steep density profiles with $k_{\rho} \approx-3$, which form because of depletion. This density exponent analysis reveals diverse density structures in a molecular cloud, forming a coherent picture that gravitational collapse and accretion contribute to a continued steepening of the density profile. We expect our method to be effective in studying other power-law-like density structures, including the density structure of granular materials and the Large-Scale Structure of the Universe.
\end{abstract}

\section{Introduction}

Many astrophysical processes, including the gravitational collapse of molecular clouds, are complex and multi-scaled. Residing in the Galactic disk, the clouds are the nurseries of stars. They are open systems that interact with the environment constantly. Their collapses involve an interplay between turbulence [1], gravity, magnetic field [2], ionization radiation, and Galactic shear, resulting in highly complex density distributions.

A clear picture of how the collapse occurs is yet to be achieved. Modern, high spatial dynamical range observations provide maps that contain an unprecedented amount of information [3]. Measures like density (Probability Distribution Function) PDF [4], correlation function [5] and fractal dimension [6] have been proposed to quantify the structure of the star-forming regions. Despite their successes, these measures are degenerate, where original data from regions are often "compressed" into 1D curves to enable subsequent analyses. The loss of information during the compression means that the complexity of the underlying structures is often overlooked. Besides, to derive these measures, one needs to specify the boundary of a region in advance, which can be a challenging task, especially for data that contain heterogeneous structures.

Power-law density structures such as $\rho \propto r^{k_{\rho}}\left(k_{\rho}<0\right)$ are common in systems which have reached intermediate asymptotic states, such that the behaviors are independent of the details of the initial and/or boundary conditions [7,8]. One such example is the gravitational collapse of molecular cloud, where stationary collapse leads to $\rho \sim r^{-2}[9,10,11,12,13]$, and accretion flow around dense objects have $\rho \sim r^{-1.5}[14,15]$. For these systems, measuring the power-law density exponent $k_{\rho}$ would enable us to distinguish different structures, and the value of $k_{\rho}$ can be directly compared against models to achieve understandings. Various attempts have been made to measure the density exponent. The most obvious approach is to fit spherical models to observational data. However, as the majority of regions 


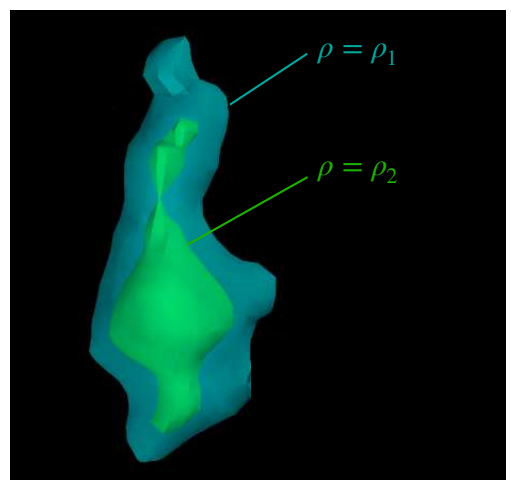

\section{Model:}

$$
\rho \approx \rho_{0}\left(r / r_{0}\right)^{k_{\rho}}
$$

Radii:

$$
\begin{aligned}
& r_{1}=\left(V_{1}\right)^{1 / 3} \\
& r_{2}=\left(V_{2}\right)^{1 / 3}=\left(V_{2,1}+V_{2,2}\right)^{1 / 3}
\end{aligned}
$$

Density exponent

$$
k_{\rho}=\frac{\log \left(\rho_{2}\right)-\log \left(\rho_{1}\right)}{\log \left(r_{2}\right)-\log \left(r_{1}\right)}
$$

Figure 1: Evaluation the Level-Set Density Exponent. We first divide a region using a set of isosurfaces. A typical region $R_{1}$ would be surrounded by an isosurface at $\rho=\rho_{1}$. Inside this region, there exists one or a few subregions $\left(R_{2, i}\right)$ surrounded by isosurfaces at $\rho=\rho_{2}$. The equations on the right hand side describe how the density exponent $k_{\rho}$ at voxels included in $R_{1}$ yet not included in $R_{2, i}$ ) is computed. The spacing between the adjacent isosurfaces are exaggerated for a clearer view.

we study are non-spherical, this approach is limited in practice. Another way is to derive the power-law density exponent using the density probability distribution function (PDF) [10, 16, 17]. Although the procedure is straightforward, this statistical approach only allows for the derivation of an "effective" density exponent, which contains no information on how gas organizes spatially.

To fully exploit the diagnostics power of the density exponent $k_{\rho}$, importing the Level-Set Method (LSM), we propose a new formalism to measure its value for non-spherical yet centrally condensed regions. The Level-Sets are contourlines, and the Level-Set Method is a conceptual framework where analyses of surfaces and shapes can be performed with the help of Level-Sets. By applying the LSM to state-of-the-art high dynamical range observations of star-forming regions, we obtain spatially-resolved maps of the density exponent, and reveal, for the first time, the complexity and regularity of molecular cloud structures.

\section{Method}

For a spherical system, the density structure can be described as $\rho(r)$ where $r$ is the radius. To measure the steepness of the density profile in a given location, we adopt a local model where $\rho \sim r^{k_{\rho}}$, and in the vicinity of $r$, the value of $k_{\rho}$ can be derived as

$$
k_{\rho}(r)=\frac{\log \left(\rho\left(r+\delta_{r}\right)\right)-\log (\rho(r))}{\log \left(r+\delta_{r}\right)-\log (r)},
$$

where $\delta_{r}<<r$.

The goal is to measure the density exponent for clouds of arbitrary geometries. Assuming a 3D density structure $\rho(x, y, z)$, we divide the region using a set of densely-spaced iso-density contours, after which each subregion should be surrounded by a contour at $\rho=\rho_{1}$, one or a few contours at $\rho=\rho_{2}$, and it should contain values ranging from $\rho_{1}$ to $\rho_{2}$. Assuming that the region $R_{1}$ has a volume of $V_{1}$, inside this region, there can be a few $(n)$ subregions $R_{2, i}$ surrounded by isosurfaces with $\rho=\rho_{2}\left(\rho_{2}>\rho_{1}\right)$, and these subregions have volumes of $V_{2, i}=V_{2,1}, \ldots V_{2, n}$. The size of the region can be approximated as $r_{1} \propto\left(V_{1}\right)^{1 / 3}$, and the effective size of all subregions altogether can be approximated as $r_{2} \propto\left(\sum_{i} V_{2, i}\right)^{1 / 3}$. The Level-Set Density Exponent is

$$
k_{\rho}=\frac{\log \left(\rho_{2}\right)-\log \left(\rho_{1}\right)}{\log \left(r_{2}\right)-\log \left(r_{1}\right)} .
$$

The procedure is illustrated in Fig. 1, and the resulting map is called the Density Exponent Map.

The advantage of the Density Exponent Analysis lies in its robustness and resolving power: the method is directly applicable to maps that contain heterogeneous structures and can be used to distinguish these structures. As an example (Fig. 2), we construct a model which contains two spherical clumps of different density profiles. We derive its density PDF and produce a density exponent map. The density PDF contains limited information since the spatial information is lost completely. In contrast, the Density Exponent Map indicates that the map should be separated into regions characterized by different density exponents and the map contain values of the density exponent at every location. The 

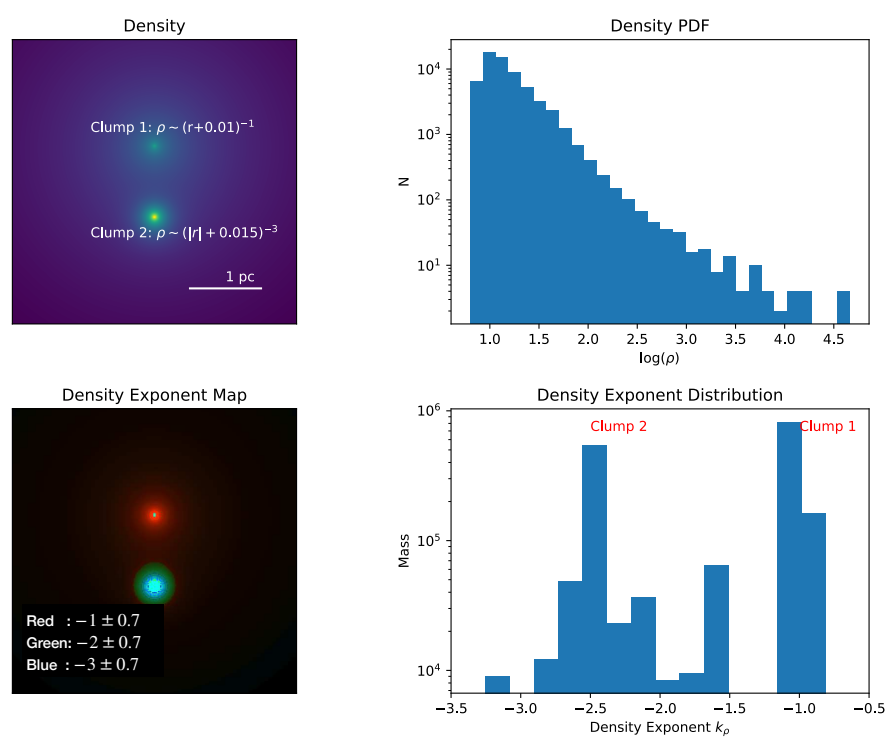

Figure 2: Methods for analyzing cloud structure. We consider an example made of two clumps (clump 1: $\rho \sim(r+0.01)^{-1}$, clump 2: $\left.\rho \sim(r+0.015)^{-3}\right)$. Upper Left: Density distribution. Upper Right: Density PDF. Lower Left: Density Exponent Map. Color represents the density exponent, and brightness represents surface density. The colormap is the same as the one in Fig. 3. Lower Right: massweighed density exponent distribution. The normalization of the $y$-axis is arbitrary.

additional spatial information retrieved by the method makes it a powerful tool to analyze complex, spatially inhomogeneous datasets, such as the density structures of nearby molecular clouds.

\section{Results \& Discussions}

Located at a distance of $\sim 290 \mathrm{pc}$ [18], the Perseus star-forming region is nearby and well-resolved. We use the surface density map derived using data from the Herschel and the Planck telescope [3]. The cloud has a size of $\sim 30 \mathrm{pc}$, and the map has a resolution of $36 \operatorname{arcsec}(\sim 0.05 \mathrm{pc})$. The enormous $\left(\sim 10^{3}\right)$ spatial dynamical range allows detailed studies.

Derivation of the level-set density exponent $k_{\rho}$ requires $3 \mathrm{D}$ density distributions. As observations are done mostly in 2D, we develop a method (see Method A) to construct 3D density distributions using $2 \mathrm{D}$ maps. This is achieved by first decomposing a $2 \mathrm{D}$ surface density map into component maps that contain structures of different sizes and by assigning thicknesses to these component maps and combining them. For cloud-like density structures, the reconstruction allows us to measure the mass-weighted mean density exponent to an accuracy of $\lesssim 0.1$. In our calculations, we focus on gas with $\rho_{\mathrm{H}_{2}}>1000 \mathrm{~cm}^{-3}$. This corresponds to $40 \%$ of the gas contained in the Perseus region and the region is surrounded by a diffuse envelope that contains gas that does not contribute directly to the star formation. We also excluded unresolved regions - patches surround by contours whose sizes are smaller than 3 pixels (0.08 pc), from our analyses. To derive the density exponent map, the data is divided using 100 contour levels equally spaced in $\log (\rho)$.

In Fig. 3 we plot the density exponent distribution at the cloud center plane, which contains all the line-of-sight density maximums. $k_{\rho}$ ranges from -3.5 to -0.5 , forming a highly inhomogeneous pattern which contain variations on different scales.

\subsection{Inter-regional variations: Density-driven collapse}

The Perseus clouds can be separated into a few pc-sized subregions. Each region has a corresponding gravitational potential dip [19], and tehse regions can collapse to from star clusters or associations. We first divide the cloud into these regions and evaluate parameters including the mass-weighed mean density exponent and the mean densities. We also characterize these regions by deriving a quantify called the dense gas fraction (see Methods B). Since all the dense gas would collapse to form individual or multiple stars, the dense gas mass fraction is a direct indicator for the star formation activity.

Correlations between these quantities are summarized in the right panel of Fig. 3, where the detailed results from the individual regions are contained in the Method $(\mathrm{C})$ section. In general, regions of steeper density profiles have higher dense gas mass fractions. Thus, the formation of dense gas, which occurs on tiny scales, is deeply linked to a global steepening of the density profile.

By plotting the mass-weighted density exponent against the mean density (Fig. 3), we find that regions of higher densities tend to have steeper density profiles, which suggests a density-driven collapse 


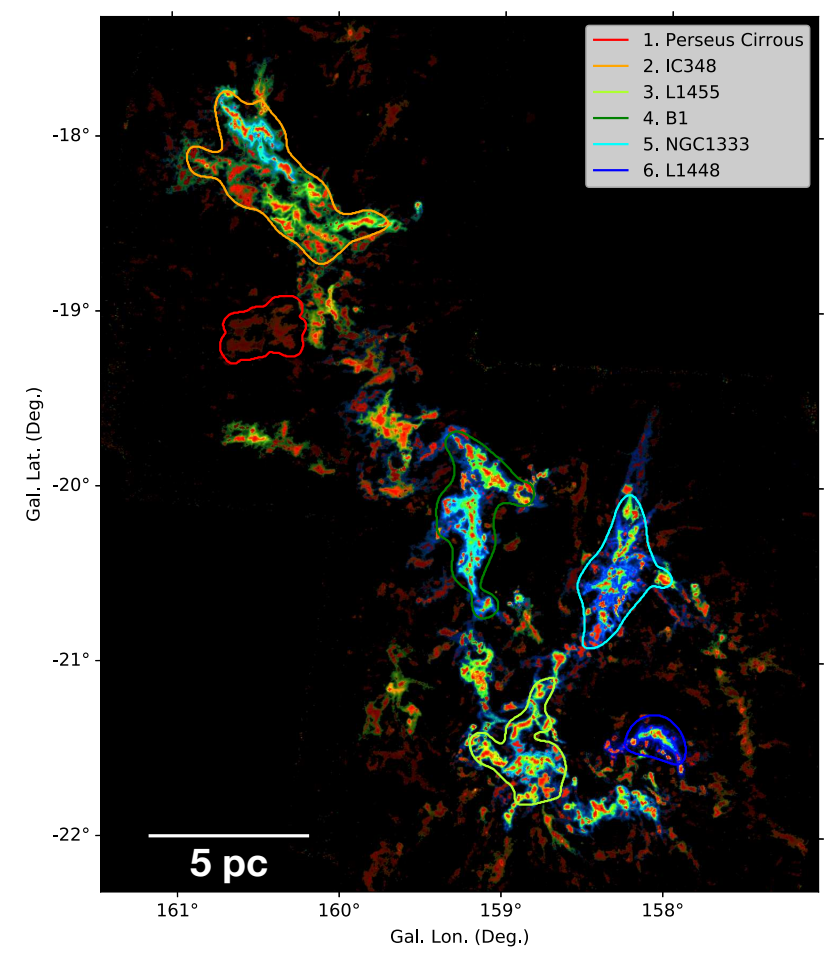

\section{Colormap}

Blue: $-3 \pm 0.7$ Green: $-2 \pm 0.7$ Red: $-1 \pm 0.7$
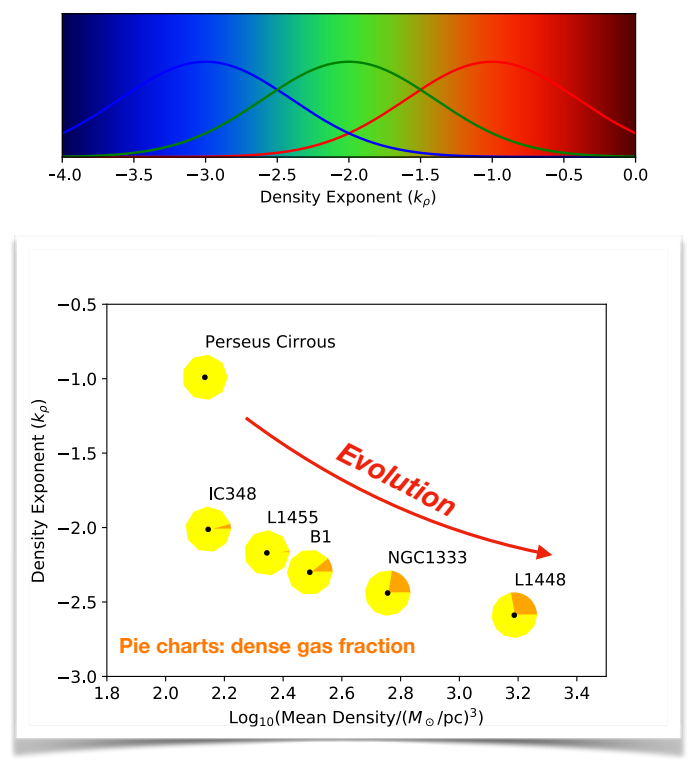

Figure 3: Results from the Perseus molecular cloud. Left: Density Exponent Map of the Perseus region. We plot the results at the midplane of the reconstructed 3D density distribution. The brightness represents the density, and the colors represent the density exponent $k_{\rho}$. The color channels have response functions of Gaussian shapes (see the colormap on the upper right). Right: Density exponent plotted against the mean density. Pie charts show the dense gas mass fractions of the corresponding regions, where the yellow areas stand for the diffuse gas and the orange areas stand for the dense gas. 
IC 348

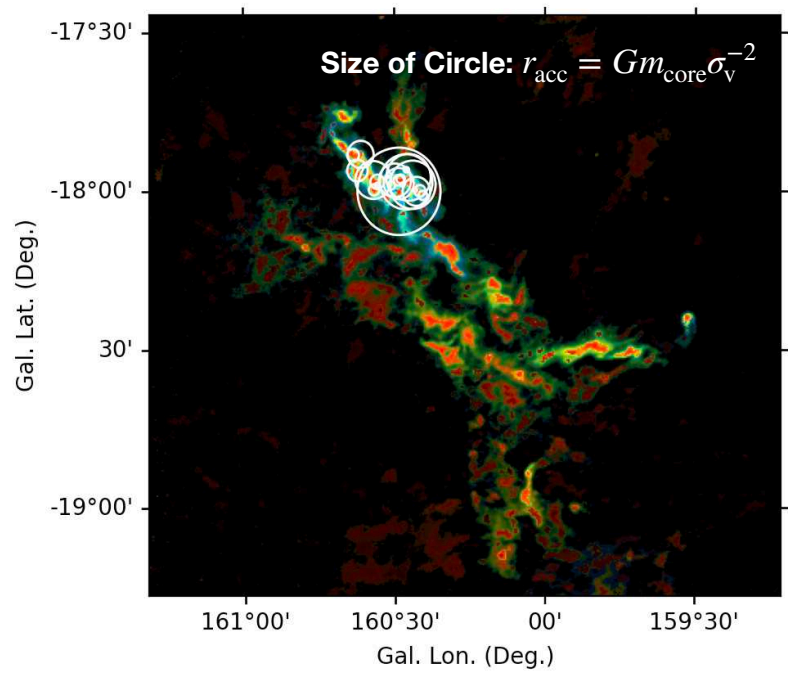

NGC1333

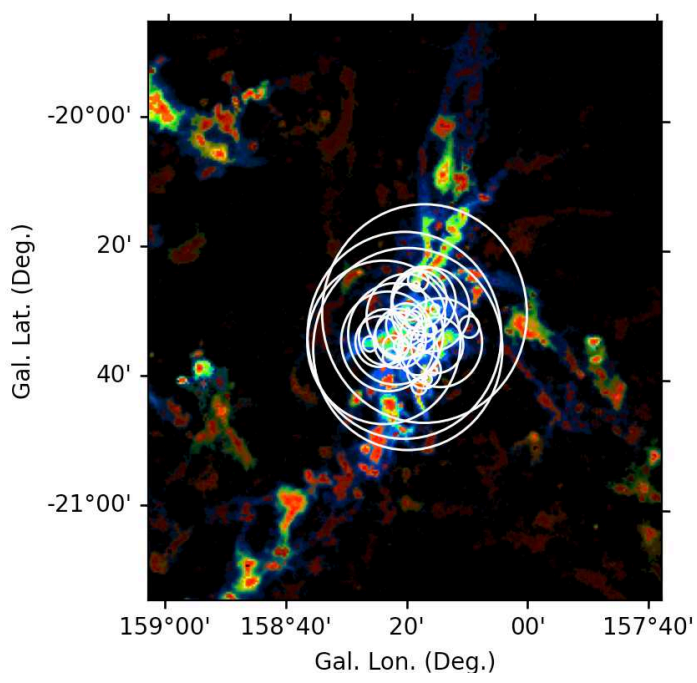

Figure 4: Stratified density structures of two subregions. In the background, we plot the density exponent map of the IC348 region and the NGC1333 region, respectively. The centers of the circles represent to the location of dense cores, and the radii of the circles correspond to the accretion zone radii of the dense cores $r_{\text {acc }}=G m_{\text {core }} / \sigma_{\mathrm{v}}^{2}$. The colormap is the same as that used in Fig. 3 .

scenario. Since all regions belong to the Perseus molecular cloud, we assume that they have almost the same age $t_{\text {Perseus. }}$ Provided that they evolve at paces set by their free-fall time $t_{\mathrm{ff}}$, which is related to the mean densities by $t_{\mathrm{ff}} \approx \sqrt{1 /(G \rho)}$, the evolutionary stage can be parameterized using $f=t_{\text {Perseus }} / t_{\mathrm{ff}} \sim$ $\rho^{1 / 2}$, and regions with larger $\rho$ are more evolved and thus their steeper density profiles and higher dense gas fractions. The picture of a steepening density profile is consistent with findings from some recent simulations [20], and the correlations point to a simple picture where the mean gas density sets the pace at which a region evolves.

The map also allows us to identified a new region called "Perseus Cirrous" (Fig. 3) for the first time. Although overlooked by previous studies, the region stands out in our analyses due to its shallow density profile $\left(k_{\rho} \approx-1\right)$. The region occupies the shallow end of the density exponent parameter space, and its structure should be representative of the structure gas at early stages of gravitational collapse.

\subsection{Intra-regional stratification: Gas depletion by accretion}

The density exponent map also reveals stratified density structures inside individual regions. In evolved regions such as the NGC1333, dense, filamentary structures of steep density profiles $\left(k_{\rho} \approx-2\right)$ are surrounded by gas of even steeper density profiles $\left(k_{\rho} \approx-3\right)$. In previous studies, regions are usually described using single power-laws [16, 17]. From our maps, we concluded that these density structures are more complex than previously believed, where multiple power-law are required to describe them.

According to theoretical studies, $k_{\rho}=-2$ represents the limiting case of stationary collapse [12]. These even steeper density profiles $\left(k_{\rho} \approx-3\right)$ demand an explanation. We propose that they are likely the result of gas depletion caused by accretion. To verify this, following the approach adopted in a previous paper [21], in Fig. 4 we plot the accretion zones of the dense cores. These zones are defined as $r<r_{\text {acc }}=G m_{\text {core }} / \sigma_{\mathrm{v}}^{2}$, where $r$ is the distance from the dense core, $m_{\text {core }}$ is the core mass, and $\sigma_{\mathrm{v}}$ is the velocity dispersion of the ambient medium. Gas located within these zones is expected to get accreted by the dense cores. The dense core catalogue is taken from literature [22], and we adopt $\sigma_{\mathrm{v}}=1 \mathrm{~km} / \mathrm{s}$, which corresponds to the velocity dispersion measured at $\sim 1 \mathrm{pc}$ scale [23]. Since the radii of the accretion zones of the majority of the cores are smaller than $1 \mathrm{pc}$, where the velocity dispersions can be smaller, we might be under-estimating the accretion radii in most cases.

We find that for regions such the NGC1333, the accretion zones of different cores overlap very significantly, meaning that these cores are accreting competitively. Remarkably, these overlapping accretion zones appear to coincide with the presence of very steep density exponents $\left(k_{\rho} \approx-3\right)$, suggesting that gas depletion by competitive accretion is the cause of the steep density profiles. 


\subsection{Summary \& Future Extensions}

The evolution of molecular clouds is exemplary of complex, multi-scale processes. Regions in molecular clouds appear to be gravitationally bound at $\sim$ pc scale [19], and the collapse of dense cores, which is directly related to star formation, occurs at $\lesssim 0.05$ pc. Importing the Level-Set Method, we develop a new, robust formalism to compute spatially resolved maps of the density exponent. On the pc scale, the mass-weighted mean density exponent correlates with the star formation activity. On smaller scales $(\lesssim$ $1 \mathrm{pc}$ ), the density exponent $k_{\rho}$ still exhibits significant variations. This complex pattern results from a continued steepening of the density profile driven by gravitational collapse and accretion.

The spatial information our method provides is valuable for large, inhomogeneous datasets. The Level Set-based formalism can be modified to suit different models, for example, to derive the scale length for exponential-like structures, which we will explore in the future. We expect our method to be effective for other structures including the density structure of granular materials and the Large-Scale Structure of the Universe.

\section{References}

[1] Mac Low, M.-M. \& Klessen, R. S. Control of star formation by supersonic turbulence. Reviews of Modern Physics 76, 125-194 (2004). astro-ph/0301093.

[2] Li, H. B. et al. The Link Between Magnetic Fields and Cloud/Star Formation. In Beuther, H., Klessen, R. S., Dullemond, C. P. \& Henning, T. (eds.) Protostars and Planets VI, 101 (2014). 1404.2024.

[3] Zari, E., Lombardi, M., Alves, J., Lada, C. J. \& Bouy, H. Herschel-Planck dust optical depth and column density maps. II. Perseus. A\&A 587, A106 (2016). 1511.08503.

[4] Kainulainen, J., Beuther, H., Henning, T. \& Plume, R. Probing the evolution of molecular cloud structure. From quiescence to birth. A\&A 508, L35-L38 (2009). 0911.5648.

[5] Padoan, P., Goodman, A. A. \& Juvela, M. The Spectral Correlation Function of Molecular Clouds: A Statistical Test for Theoretical Models. ApJ 588, 881-893 (2003). astro-ph/0211135.

[6] Hetem, J., A. \& Lepine, J. R. D. Fractal 3-D simulations of molecular clouds. A\&A 270, 451-461 (1993).

[7] Barenblatt, G. I. Scaling, Self-similarity, and Intermediate Asymptotics (1996).

[8] Goldenfeld, N., Martin, O. \& Oono, Y. Intermediate asymptotics and renormalization group theory. Journal of Scientific Computing 4, 355-372 (1989). URL https://doi.org/10.1007/BF01060993.

[9] Kritsuk, A. G., Norman, M. L. \& Wagner, R. On the Density Distribution in Star-forming Interstellar Clouds. ApJ 727, L20 (2011). 1007.2950.

[10] Girichidis, P., Konstandin, L., Whitworth, A. P. \& Klessen, R. S. On the Evolution of the Density Probability Density Function in Strongly Self-gravitating Systems. ApJ 781, 91 (2014). 1310.4346.

[11] Naranjo-Romero, R., Vázquez-Semadeni, E. \& Loughnane, R. M. Hierarchical Gravitational Fragmentation. I. Collapsing Cores within Collapsing Clouds. ApJ 814, 48 (2015). 1510.05617.

[12] Li, G.-X. Scale-free gravitational collapse as the origin of $\rho \sim \mathrm{r}^{-2}$ density profile - a possible role of turbulence in regulating gravitational collapse. MNRAS 477, 4951-4956 (2018). 1803.03273.

[13] Donkov, S. \& Stefanov, I. Density distribution function of a self-gravitating isothermal compressible turbulent fluid in the context of molecular clouds ensembles - II. Contribution of the turbulent term and the potential of the outer shells. MNRAS 485, 3224-3229 (2019). 1903.01903.

[14] Hoyle, F. \& Lyttleton, R. A. On the accretion theory of stellar evolution. MNRAS 101, 227 (1941).

[15] Bondi, H. On spherically symmetrical accretion. MNRAS 112, 195 (1952).

[16] Li, G.-X. \& Burkert, A. Constructing multiscale gravitational energy spectra from molecular cloud surface density PDF - interplay between turbulence and gravity. MNRAS 461, 3027-3035 (2016). 1603.04342. 
[17] Parmentier, G. \& Pasquali, A. A New Parameterization of the Star Formation Rate Dense Gas Mass Relation: Embracing Gas Density Gradients. ApJ 903, 56 (2020). 2009.10652.

[18] Zucker, C. et al. Mapping Distances across the Perseus Molecular Cloud Using CO Observations, Stellar Photometry, and Gaia DR2 Parallax Measurements. ApJ 869, 83 (2018). 1803.08931.

[19] Li, G.-X., Wyrowski, F., Menten, K., Megeath, T. \& Shi, X. G-virial: Gravity-based structure analysis of molecular clouds. A\&A 578, A97 (2015). 1504.01003.

[20] Gómez, G. C., Vázquez-Semadeni, E. \& Palau, A. Density profile evolution during prestellar core collapse: Collapse starts at the large scale. arXiv e-prints arXiv:2009.14151 (2020). 2009.14151.

[21] Li, G.-X., Cao, Y. \& Qiu, K. Network of Star Formation: Fragmentation controlled by scaledependent turbulent pressure and accretion onto the massive cores revealed in the Cygnus-X GMC complex. arXiv e-prints arXiv:2107.00870 (2021). 2107.00870.

[22] Curtis, E. I. \& Richer, J. S. The properties of SCUBA cores in the Perseus molecular cloud: the bias of clump-finding algorithms. MNRAS 402, 603-619 (2010). 0910.4070.

[23] Larson, R. B. Turbulence and star formation in molecular clouds. MNRAS 194, 809-826 (1981).

[24] Clark, P. C., Glover, S. C. O., Ragan, S. E. \& Duarte-Cabral, A. Tracing the formation of molecular clouds via [C II], [C I], and CO emission. MNRAS 486, 4622-4637 (2019). 1809.00489.

[25] Enoch, M. L. et al. Bolocam Survey for $1.1 \mathrm{~mm}$ Dust Continuum Emission in the c2d Legacy Clouds. I. Perseus. ApJ 638, 293-313 (2006). astro-ph/0510202.

Methods:

\section{A Reconstruction of 3D density structure}

We develop a formalism to construct 3D density structures from $2 \mathrm{D}$ observations. The reconstruction consists of two steps. First, using a method called "constrained diffusion decomposition" (Li 2021 ApJS under revision), we decompose the surface density maps into component maps that contain structures of different sizes. The first of these maps contain structures whose sizes range from 1 to 2 pixels, and the $n$th of these maps contain structures of sizes between $2^{n-1}$ and $2^{n}$ pixels. Second, a 3D density structure is constructed using the component maps. During our reconstruction, the channels are assumed to be slabs of different thicknesses, where and along the line of sight, the $n$th channel has a Gaussian density profile of dispersion of $2^{n}$ pixels. The final 3D density structure is assumed to be the sum of these slabs. When combining these slabs, we aligned them such that the density maximums stay on the same plane.

Due to the lack of information on the distribution of gas along the line of sight direction, the density structure we constructed is not identical to but resembles the real one. We first test our method by producing a 3D clump of where $\rho \sim r^{-2}$, projected it to 2D, and verified that our reconstruction allows us to recover the density exponent to an accuracy of $\lesssim 0.01$. Then, using results from numerical simulations [24], we perform density exponent analysis on both the original data and the 3D data constructed from a $2 \mathrm{D}$ projection. We limit ourselves to gas with $n\left(\mathrm{H}_{2}\right) \gtrsim 250 \mathrm{~cm}^{-3}$ and find that the reconstructed cloud and the original cloud are similar in terms of $k_{\rho}$ (Fig. 5). The original cloud has a mass-weighted density exponent of -1.66 , and the reconstructed cloud has $k_{\rho}=-1.69$. The difference is noticeable but is still small compared to the variations we are interested in. Although some small-scale details are lost, compared to the original cloud, the reconstructed cloud has very similar density exponent distributions.

\section{B Measurement of dense gas fraction and star formation activ- ity}

The star formation activity is characterized by the dense gas ratio - the ratio between the amount of the dense gas which should collapse to form stars and the total amount of gas in a region. To trace the dense gas, we use the $870 \mu \mathrm{m}$ observation towards the Perseus region where only dense gas can be observed [25], and created a mask containing significant $(\gtrsim 3.5 \sigma)$ detections. Using the Herschel-Planck surface density map [3], the total amount of gas is derived by integrating over whole regions, and the amount of dense gas is derived by integrating over subregions with significant Bolocam $870 \mu \mathrm{m}$ detections. 


\section{Simulation}

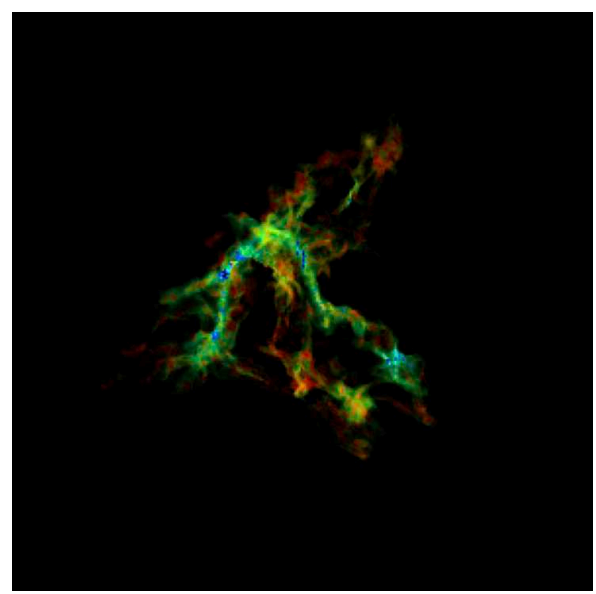

Reconstructed

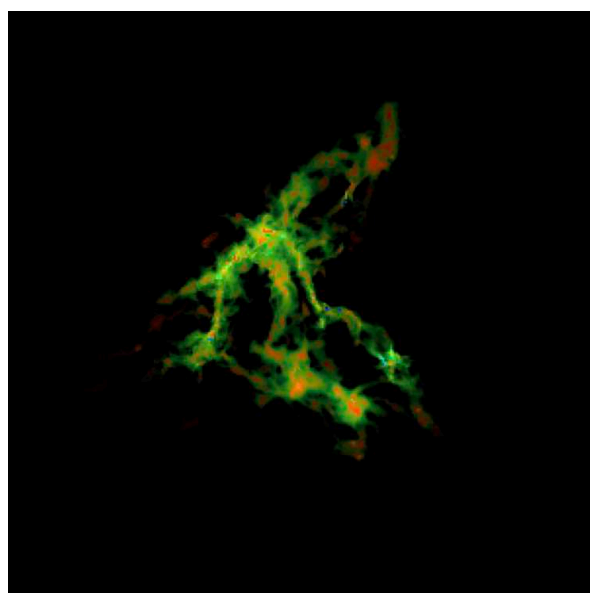

Figure 5: Testing the 3D density reconstruction method. Left panel: Map of mass-weighted mean density exponent of a simulated cloud. Right panel: Map of mass-weighted mean density exponent of the reconstructed cloud. The brightness represents the density, and the color represents the density exponent. Red: $k_{\rho}=-1 \pm 0.7$, green: $k_{\rho}=-2 \pm 0.7$, and blue: $k_{\rho}=-3 \pm 0.7$.

\section{Results from all subregions}

In Fig. 6 we plot results from all subregions. These plots are arranged according to their evolutionary stages inferred from the mass-weighed mean density exponent. Regions in the bottom panels are more evolved compared to regions in the top panels. The amount of unresolved gas - gas contained in regions whose sizes are smaller than three pixels $\left(56^{\prime \prime}, 0.08 \mathrm{pc}\right)$, are also indicated. 

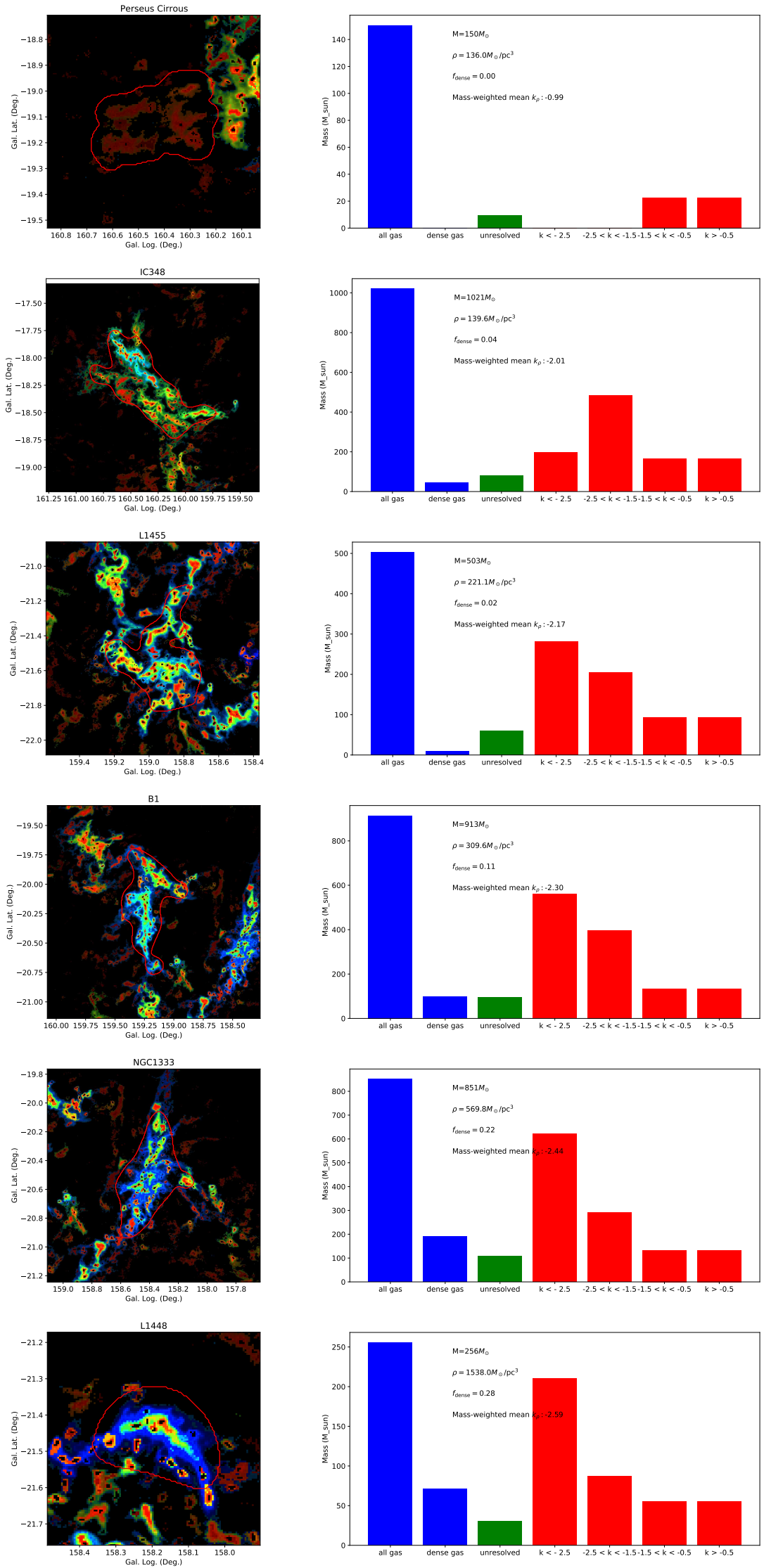

Figure 6: Results from individual regions. Left panels: maps of the density exponent $k_{\rho}$. Right panels: we plot the total amount of gas, the amount of dense gas, the amount of spatially-unresolved gas, as well as a distribution of gas in regions of different $k_{\rho}$. Properties including total gas mass, mean density, dense gas fraction, and mass-weighted density exponent, are indicated in the right panels. Limited by the resolution, it is impossible to derive the density exponent for gas contained in contours whose sizes are smaller than 3 pixels. Mass contained in these contours are excluded from the analyses. The total amount of unresolved gas are indicated as "unresolved". 